\title{
Discretization and Grouping: Preprocessing Steps for Data Mining
}

\author{
Petr Berka ${ }^{1}$ and Ivan Bruha ${ }^{2}$ \\ ${ }^{1}$ Laboratory of Intelligent Systems \\ Prague University of Economic \\ W. Churchill Sq. 4, Prague CZ-13067, Czech Republic \\ email: berka@vse.cz \\ ${ }^{2}$ Department of Computer Science and Systems, McMaster University \\ Hamilton, Ont., Canada L8S4K1 \\ email: bruha@mcmaster.ca
}

\begin{abstract}
Unlike on-line discretization performed by a number of machine learning (ML) algorithms for building decision trees or decision rules, we propose off-line algorithms for discretizing numerical attributes and grouping values of nominal attributes. The number of resulting intervals obtained by discretization depends only on the data; the number of groups corresponds to the number of classes. Since both discretization and grouping is done with respect to the goal classes, the algorithms are suitable only for classification/prediction tasks.

As a side effect of the off-line processing, the number of objects in the datasets and number of attributes may be reduced.

It should be also mentioned that although the original idea of the discretization procedure is proposed to the Kex system, the algorithms show good performance together with other machine learning algorithms.
\end{abstract}

\section{Introduction}

The Knowledge Discovery in Databases (KDD) process can involve a significant iteration and may contain loops among data selection, data preprocessing, data transformation, data mining, and interpretation of mined patterns. The most complex steps in this process are data preprocessing and data transformation. The result of these steps should be data in the form suitable for the data mining algorithms used ${ }^{1}$. Thus, the necessary preprocessing and transformation operations are e.g. "merging" data to create singe data matrix from a number of related data tables, creating new attributes, excluding unnecessary attributes, discretization or grouping.

We present here algorithms for the last two mentioned operations: "off-line" discretization of numerical attributes and "off-line" grouping values of nominal attributes. Such transformations can help in better understanding the data that

${ }^{1}$ If we insist on interpretability of the discovered knowledge, we should prefer symbolic machine learning algorithms. 
enter into the data mining step. Both algorithms are class-sensitive, so they can be used only for classification/prediction tasks.

The described algorithms were developed within the KEx framework [2] but can be used as a preprocessing tool for other ML algorithms as well.

\section{Discretization}

The task of discretization of numerical variables is well known to statisticians. Different approaches are used such as discretization into a given number of categories using equidistant cutpoints, or discretization based on mean and standard deviation. All these approaches are "class-blind", since they do not take into account that objects belong to different classes. Hence they can be used if the data mining algorithms perform unsupervised learning ${ }^{2}$. If the task is classification/prediction the information about the class label can be used during discretization.

In the TDIDT family, the algorithms for discretization are based mostly on binarization within a subset of training data created during tree generation [6]. Well known algorithm by Fayyad and Irani extended this idea to multi-interval discretization [8]. KnowledgeSeeker, a commercial system of the TDIDT family, looks for "best" k-way split of each variable using $F$ statistics for numerical attributes and $\chi^{2}$ statistic for nominal attributes; so the system performs both discretization and grouping [4].

Discretization in the set covering algorithm CN4 (a large extension of CN2) is based on entropy or Laplacian estimate [5].

All these systems discretize numerical attributes "on-line", during learning. The algorithms described in the paper preprocess the data "off-line" before starting the machine learning step.

\section{Discretization and grouping for KEX}

\subsection{A survey of the system}

KEX performs symbolic empirical multiple concept learning from examples, where the induced concept description is represented as weighted decision rules in the form

$$
\text { Ant } \Longrightarrow C \text { (weight) }
$$

where $A n t$ is a combination (conjunction) of attribute-value pairs,

$C$ is a single category (class),

weight from the interval $[0,1]$ expresses the uncertainty of the rule.

KEX [2] works in an iterative way, in each iteration testing and expanding an implication $A n t \Longrightarrow C$. This process starts with an "empty rule" weighted

${ }^{2}$ This case is carried out when the data mining task is to find some interesting groups of objects with similar characteristics or to find associations. 
with the relative frequency of the class $C$ and stops after testing all implications created according to the user defined criteria. During testing, the validity (conditional probability $P(C \mid A n t)$ ) of an implication is computed ${ }^{3}$. If this validity significantly differs from the composed weight (value obtained when composing weights of all subrules of the implication $A n t \Rightarrow C$ ), then this implication is added to the knowledge base. The weight of this new rule is computed from the validity and from the composed weight using inverse composing function. For composing weights we use a pseudobayesian (Prospector-like) combination function

$$
x \oplus y=\frac{x * y}{x * y+(1-x) *(1-y)} .
$$

During expanding, new implications are created by adding single categories to Ant.

\subsection{Algorithm for discretization}

We treat each numerical attribute separately. The basic idea is to create intervals for which the aposteriori distribution of classes $P(C \mid$ interval $)$ significantly differs from the apriori distribution of classes $P(C)$ in the whole training data. This can be achieved by simply merging such values, for which most objects belong to the same class. Within the KEx knowledge acquisition approach, this will lead to rules of the form

$$
\text { interval } \Longrightarrow C
$$

but this approach can be used for other learning algorithms, too.

The number of resulting intervals is "controlled" by giving a threshold for minimal number of objects within one interval; less frequent intervals are labeled as "UNKNOWN" in the step $\mathbf{3 . 1}$ (the algorithm is shown in Figure 1).

\subsection{Algorithm for grouping}

Grouping values of a nominal attribute becomes important if the number of these values is too large (e.g. hundreds of ZIP codes or profession codes). To deal with each value separately can bring problems both during computation (e.g. branching a node) and interpretation.

The grouping algorithm for KEX is based on the same idea as the discretization described above. Again, we create groups of values such, that the aposteriori distribution $P(C \mid$ group $)$ significantly differs from the apriori distribution $P(C)$. The main difference to the discretization algorithm is that we create single group for each value of class attribute and an additional group labeled "UNKNOWN"; so the number of groups is fixed. The grouping algorithm is shown in Figure 2.

\footnotetext{
${ }^{3}$ The validity $P(C \mid A n t)$ is computed from the data as $\frac{\|A n t n C\|}{\|A n t\|}$.
} 


\section{MAIN LOOP:}

1 create ordered list of values of the attribute;

2 for each value do

2.1 compute frequencies of occurrence of objects with respect to each class;

2.2 assign the class label to every value using procedure ASSIGN; enddo

3 create the intervals from values using procedure INTERVAL;

\section{ASSIGN:}

if for the given value all objects belong to same class, then assign the value to that class

else if for the given value the distribution of objects with respect to class membership significantly differs (according to $\chi^{2}$ or relative frequency criterion) from frequencies of goal classes, then assign that value to the most frequent class

else assign the value to the class "UNKNOWN";

\section{INTERVAL:}

3.1 if a sequence of values belongs to the same class, then create the interval $I N T_{i}=\left[\right.$ LBound $_{i}, U$ Bound $\left._{i}\right]$ from these values;

3.2 if the interval $I N T_{i}$ belongs to the class "UNKNOWN" then

if its neighbouring intervals $I N T_{i-1}, I N T_{i+1}$ belong to the same class then create the interval by joining $I N T_{i-1} \cup I N T_{i} \cup I N T_{i+1}$

else create the interval either by joining $I N T_{i-1} \cup I N T_{i}$ or by joining $I N T_{i} \cup$ $I N T_{i+1}$ according to given criterion (For $\chi^{2}$ test join the intervals according to the higher value of $\chi^{2}$, for frequency criterion join the intervals according to higher relative frequency of the majority class.);

3.3 create continuous coverage of the attribute by assigning LBound; := (LBound $_{i}+U$ Bound $\left._{i-1}\right) / 2$ and $U$ Bound $_{i-1}:=$ LBound $_{i}$;

Fig. 1. Algorithm for discretization

\section{MAIN LOOP:}

1 for each value do

1.1 compute frequencies of occurrence of objects with respect to each class;

1.2 assign the class label to every value using procedure ASSIGN; enddo

2 create the groups from values using procedure GROUP;

\section{ASSIGN:}

if for the given value all objects belong to same class, then assign the value to that class

else if for the given value the distribution of objects with respect to class membership significantly differs (according to $\chi^{2}$ or relative frequency criterion) ) from frequencies of goal classes, then assign that value to the most frequent class

else assign the value to the class "UNKNOWN";

\section{GROUP:}

3.1 create groups for values with the same class label; 


\subsection{Evaluation of the algorithms}

During discretization/grouping of an attribute, we can loose some information hidden in the data. We can measure this loss by the number of contradictions before and after the preprocessing. Contradiction here means a situation when objects described by same attribute values belong to different classes. Any learning algorithm will classify such objects as objects belonging to the same (usually the majority) class and objects belonging to other classes will be classified wrongly. We can count such errors and thus estimate the maximal possible accuracy as:

$$
1-\frac{\text { no. of errors }}{\text { no. of objects }}
$$

As a side effect of the off-line processing, the number of objects in the datasets and number of attributes may be reduced. This reduction can give us some information about regular patterns in the data.

Another useful information is the number of intervals or groups. If the preprocessing results in a single interval/group or in one interval/group with very high frequency in the data, we can ignore the corresponding attribute in the subsequent machine learning step.

\section{Empirical results}

We have tested our algorithm on different datasets taken from the UCI Machine Learning Repository [10]. The results are summarized in Table 1. This table gives for the used data sets ${ }^{4}$ the number of (different) objects and the maximal possible accuracy both before and after discretization. We present also the results of classification accuracy for different systems to show that there is no significant difference between KEx (system, that motivates the discretization algorithm) and other systems (CN4 ordered mode, CN4 unordered mode, and C4.5).

\begin{tabular}{|c|c|c|c|c|c|c|}
\hline data & \multicolumn{2}{|c|}{$\begin{array}{l}\text { accuracy \#.diff. } \\
\text { in data objects }\end{array}$} & \multicolumn{4}{|c|}{\begin{tabular}{|c} 
classification accuracy \\
KEX CN4 CN4 C4.5 \\
ord. unord.
\end{tabular}} \\
\hline credit (orig.) & $00 \%$ & 125 & - & $97 \%$ & $81 \%$ & $83 \%$ \\
\hline credit (disc.) & 100 & 117 & $97 \%$ & $95 \%$ & $88 \%$ & $76 \%$ \\
\hline iris (orig.) & $100 \%$ & 147 & - & $99 \%$ & $97 \%$ & $98 \%$ \\
\hline & $98 \%$ & 35 & $95 \%$ & $98 \%$ & $95 \%$ & $96 \%$ \\
\hline diab. ( & $100 \%$ & 768 & - & $96 \%$ & $79 \%$ & $90 \%$ \\
\hline diab. (disc.) & $95 \%$ & 538 & $85 \%$ & $84 \%$ & $81 \%$ & $75 \%$ \\
\hline & $100 \%$ & 690 & - & $100 \%$ & $87 \%$ & $91 \%$ \\
\hline aust. (disc.) & $99 \%$ & 663 & $87 \%$ & $92 \%$ & $89 \%$ & $89 \%$ \\
\hline
\end{tabular}

Table 1. Discretization of some UCI data

\footnotetext{
${ }^{4}$ Credit stands for Japanese credit data, iris stands for Iris data, diab for Pima Indian diabetes data, and aust for Australian credit data.
} 
We used our discretization/grouping algorithm also to preprocess a more complex data [3]. The KDD Sisyphus data is an excerpt from a Swiss Life data warehouse system MASY [9]. These data consist of several relations describing relationship between Swiss Life partners, insurance contracts and components of insurance tariffs. The goal of KDD on this data is to find concept description for given class (TaskA, TaskB). After creating single data matrix for each task we run the discretization and grouping algorithms for the TaskA and TaskB data. The results (in terms of maximal possible accuracy before and after this preprocessing) are shown in table 2. Discretization and grouping helped us also to find unimportant attributes; lines Taskx (disc.+red. ) in the table show the accuracy and number of objects after removing such attributes.

\begin{tabular}{|l|ccc|}
\hline data & accuracy in data & \#.diff. objects & \#. attributes \\
\hline TaskA (orig.) & $100 \%$ & 17267 & 46 \\
TaskA (disc.) & $98 \%$ & 13138 & 46 \\
TaskA (disc.+red.) & $98 \%$ & 12773 & 43 \\
TaskB (orig.) & $100 \%$ & 12945 & 83 \\
TaskB (disc.) & $100 \%$ & 12945 & 83 \\
TaskB (disc.+red.) & $100 \%$ & 12945 & 75 \\
\hline
\end{tabular}

Table 2. Discretization and grouping of KDD Sisyphus data

\section{Conclusion}

Unlike on-line discretization performed by a number of ML algorithms during building decision trees or decision rules, we propose off-line algorithms for discretizing numerical attributes and grouping values of nominal attributes. The number of resulting intervals obtained during discretization depends only on the data; the number of groups corresponds to the number of classes. Since both discretization and grouping is done with respect to the goal classes, the algorithms are suitable only for classification/prediction tasks.

The obtained intervals/groups can be interpreted as potential knowledge. The results of the off-line discretization and grouping can also help during further preprocessing of the data. If we obtain single interval, we can conclude that the discretized attribute is irrelevant for the classification task. We can draw the same conclusion also if after discretization or grouping almost all objects fall into one interval/group.

It should be also mentioned that although the original idea of the discretization procedure is related to the $\mathrm{KEX}$, the algorithms show good performance together with other machine learning algorithms. 


\section{References}

1. Berka,P. - Bruha,I.: Various discretization procedures of numerical attributes: Empirical comparisons. In: (Kodratoff, Nakhaeizadeh, Taylor eds.) Proc. MLNet Familiarization Workshop on Statistics, Machine Learning and Knowledge Discovery in Databases, Herakleion, 1995, p.136-141.

2. Berka,P. - Ivánek,J.: Automated Knowledge Acquisition for PROSPECTOR-like Expert Systems. In. (Bergadano, deRaedt eds.) Proc. ECML'94, Springer 1994.

3. Berka,P. - Sochorová,M. - Rauch,J.: Using GUHA and KEX for Knowledge DIscovery in Databases; the KDD Sisyphus Experience. In Proc: Poster Session ECML'98, TU Chemnitz 1998.

4. Biggs,D. - de Ville,B - Suen,E.: A method of choosing multiway partitions for classification and decision trees. Journal of Applied Statistics, Vol. 18, No. 1, 1991, 49-62.

5. Bruha,I. - Kočková,S.: A covering learning algorithm for cost-sensitive and noisy environments. In: Proc. of ECML'93 Workshop on Learning Robots, 1993.

6. Catlett, J.: On changing continuous attributes into ordered discrete attributes. In: Y. Kodratoff, ed.: Machine Learning - EWSL-91, Springer-Verlag, 1991, 164-178.

7. Clark,P. - Niblett,T.: The CN2 induction algorithm. Machine Learning, 3 (1989), 261-283

8. Fayyad,U.M. - Irani,K.B.: Multi-Interval Discretization of Continuous-Valued Attributes for Classifiacation Learning. In: Proc. IJCAI'93, 1993.

9. Kietz,J.U. - Reimer,U. - Staudt,M.: Mining Insurance Data at Swiss Life. In: Proc. 23rd VLDB Conference, Athens, 1997.

10. Merz,C.J. - Murphy,P.M.: UCI Repository of Machine Learning Databases. Irvine, University of California, Dept. of Information and Computer Science, 1997. 